\title{
La relación entre la academia y la investigación en la Universidad
}

Dr. José Carlos Monzón Fuentes Director de Iecis

En el ámbito internacional, muchas universidades además de su nombre propio, son llamadas «Universidades de investigación». Esto es para distinguir a aquellas que consideran a la investigación como una parte central de su misión y su quehacer universitario. Ocurre con mayor frecuencia en países de ingresos altos y estas instituciones en su mayoría son públicas, con algunas excepciones como en Estados Unidos o Japón (1). En estas instituciones existe el compromiso de la creación y diseminación de conocimiento en un rango de disciplinas y campos, que promueve el uso de sus laboratorios y desarrollan nuevas instalaciones e infraestructura que permite llevar las actividades de investigación al máximo nivel.

Sin embargo, la importancia e impacto que tienen en países de bajos o medianos ingresos como Guatemala no siempre es visualizada. En estos países, las universidades les permiten a sus estudiantes acceder al mercado del conocimiento que es cada vez más relevante para ser competitivos a nivel mundial. El área de investigación no es la excepción y aunque las universidades de investigación en estos países aún no alcanzan los rankings globales son muy importantes y están en una mejora continua (1).

Es importante reconocer que, las universidades de investigación trabajan en un contexto donde la investigación debe ser reconocida y aceptada en todo nivel, incluso el gubernamental. Existe evidencia que reporta que los gobiernos de países de bajos y medianos ingresos no priorizan la investigación, la innovación o desarrollo (2). Esto obedece a varias razones: como la existencia de un entrenamiento en investigación y financiamiento deficientes y la fuga de investigadores prominentes hacia otros países con mejores oportunidades (2), (3). Además, estos intereses pueden competir con asuntos de urgencia nacional como: salud, seguridad alimentaria o cualquier otro servicio básico, del cual carece la población. Sin embargo, es acá donde las instituciones de investigación tienen un potencial de acción amplio y esto es especialmente cierto en las ciencias de la salud, en donde la investigación, en las diferentes ramas de esta disciplina, juega un papel crucial para el abordaje de los problemas de salud en un país (4), (5).

Para lograr posicionar a la investigación al servicio de las políticas públicas, es importante establecer los patrones de financiamiento más utilizados para definir rutas administrativas que sean ágiles, que permitan la ejecución 
de fondos de manera puntual y ordenada en cualquier institución sea pública o privada. Phillipp Altbach de la Universidad de Singapur subraya que las universidades de investigación están en la cima de la jerarquía académica y que son centrales en el éxito de cualquier economía basada en el conocimiento a la que cualquier país puede acceder. En Guatemala, se necesitan de estas instituciones para participar en el ámbito globalizado de la educación superior de manera que se siga con el fomento del progreso de la sociedad. Y un paso inicial esencial es la construcción de capacidades de investigación y de gestión del financiamiento, que permite dar los primeros pasos hacia un sistema universitario sostenible. Propuestas de investigación robustas y un plan de trabajo que incluya la adecuada identificación de los costos totales de cada proyecto, esto es, costos directos e indirectos que necesita para funcionar (5), son aspectos fundamentales que las instituciones deben fomentar para permitir la creación de conocimiento a través de la investigación.

En el contexto previamente descrito, se presentan nuevas oportunidades para instituciones como la Universidad Rafael Landívar (URL) de asumir el desafío y desarrollar la investigación científica al más alto nivel. La URL, desde su fundación, ha fortalecido progresivamente su presencia como institución de educación superior en el país. Ahora, se han tomado varios pasos para resaltar la importancia de la investigación dentro del quehacer universitario, en este sentido, se estableció la Vicerrectoría de Investigación (VRIP) en donde se reafirma la decisión de la Universidad de impulsar la investigación transformadora desde el máximo 14 nivel institucional posible y de especializarla corporativamente, en la búsqueda de la

interdisciplinariedad y la

transdisciplinariedad desde la excelencia disciplinar (6).

Asimismo, de consolidar las comunidades epistémicas dentro de los Institutos de Investigación y Proyección, entre estos, entre la VRIP y entre las otras vicerrectorías del Sistema Universitario Landivariano (SUL) (6). Estas comunidades epistémicas e intercambios son los que se buscan con el plan de movilidad, tal y como lo hacen las universidades de investigación en el ámbito internacional, en donde los investigadores son profesores y estos son investigadores del más alto nivel. Esto requerirá cambios y esfuerzos institucionales, pero sin duda tendrá muchos beneficios para todo el SUL.

En este ámbito, la conexión de la VRIP con las facultades de la Vicerrectoría Académica (VRAC) se ha impulsado desde hace varios años, al menos la mitad de los investigadores de la VRIP participa en labores docentes formales en estas facultades. Asimismo, algunas de las unidades de la VRIP como el Instituto de Investigación y Estudios Superiores en Ciencias de la Salud (lecis) apoyan a 
la Facultad de Ciencias de la Salud en: la conceptualización, diseño y puesta en marcha de programas formales a nivel superior. Esta articulación con la VRAC permite la integración de la labor de investigación para dar fortaleza a todo el SUL (6) y crear ventajas competitivas al ofrecer experiencias de investigación en diversas disciplinas a las y los estudiantes y profesores universitarios.

Tal y como se describe en la Agenda de Investigación y Proyección (6): «En cuanto a los procesos formativos desarrollados por la VRIP es preciso señalar que se conciben y organizan buscando la complementariedad con los procesos de formación formal que están bajo la conducción de la VRAC, a través de las distintas facultades en campus y sedes». De hecho, al menos el $40 \%$ de los investigadores de la VRIP participan como docentes en las facultades y son varias las iniciativas de: conceptualización, diseño y puesta en marcha de programas de formación que han contado con el talento de los investigadores de la VRIP, en tanto capacidades del sistema universitario. Sin embargo, de manera más cotidiana, la formación que ofrece la VRIP es informal, en el sentido que no es titulada, aunque no por ello se aleja de las nociones landivarianas de calidad, innovación e inclusión. Conceptualmente, el mandato de la VRIP en materia de formación resulta indispensable y complementario para potenciar los alcances de la investigación y la incidencia universitaria en apego al concepto articulador de proyección (6).

A manera de conclusión, la vinculación VRIP-VRAC es un paso muy importante en el avance hacia una verdadera Universidad de investigación, donde existe en distintos ámbitos de la URL, aunque centralizada en la VRIP pero no limitada por ella.

\section{Referencias}

1. Higher Education Web Publishing Ltd. The role of research universities in developing countries. University World News. 11 de agosto de 2013. Disponible en: https://www.universityworldnews.com/post. php?story $=20130811091502202$

2. Ghaffar A, ljsselmuiden C, Zicker F. Changing Mindsets Research Capacity Strengthening in Low-and Middle-Income Countries. Council on Health Research for Development (COHRED) Global Forum for Health Research Special Programme for Research \& Training in Tropical Diseases (TDR). 2007. Disponible en: https://www.researchgate.net/publication/265432931_ CHANGING_MINDSETS_Research_capacity_strengthening_in_low-_and_ middle-income_countrie

3. Ogundahunsi OAT, Vahedi M, Kamau EM et al. Strengthening Research Capacity-TDR's Evolving Experience in Low- and Middle-Income Countries. Plos Neglected Tropical Diseases. Enero de 2015;9(1):e3380. Disponible en: https://journals.plos.org/plosntds/article?id=10.1371/journal.pntd.0003380

4. Cash-Gibson L, Guerra G, Salgado-de-Snyder VN. SDH-NET: A SouthNorth-South collaboration to build sustainable research capacities on social determinants of health in low-and middle-income countries. Health Research Policy and Systems. 2015;13(45). doi:10.1186/s12961-015-0048-1

5. Essence on Health Research. Five Keys to Improving Research Costing in Lowand Middle-Income Countries. 2012. Disponible en: https://www.who.int/tdr/ publications/TDR_ESSENCE_1.12_eng.pdf?ua $=1$

6. Vicerrectoría de Investigación y Proyección de la Universidad Rafael Landívar. Agenda de Investigación y Proyección: Documento sintético de los programas de investigación científico-críticos; 2016. Disponible en: https://www.url.edu.gt/ publicacionesurl/ppublicacion.aspx?pb=200 